\title{
CORRIGENDUM
}

\section{Developmental competence of ovine oocyte following vitrification: effect of oocyte developmental stage, cumulus cells, cytoskeleton stabiliser, FBS concentration, and equilibration time - CORRIGENDUM}

\author{
Abolfazl Shirazi, Fatemeh Taheri, Hassan Nazari, Maryam Norbakhsh-nia, \\ Ebrahim Ahmadi and Banafsheh Heidari
}

doi:10.1017/S096719941200038X, Published by Cambridge University Press, 14 August 2012.

The Authors apologise for an error in the affiliation which appears under the authors' names on the title page of this article. 'Shahid Beheshti University' should not have been included. The affiliation should have appeared as follows:-

Reproductive Biotechnology Research Center, Avicenna Research Institute, ACECR, Tehran, Iran; and Research Institute of Animal Embryo Technology, Shahrekord University, Shahrekord, Iran

And the footnote on page 1, should read as follows:-

${ }^{1}$ All correspondence to: Banafsheh Heidari. Reproductive Biotechnology Research Center, Avicenna Research Institute, ACECR, P.O. Box: 19615-1177, Tehran, Iran.

Tel: +98 21 22404144. Fax: +98 21 22432021. e-mail: ban_heidari@yahoo.com or b.heidari@avicenna.ac.ir

${ }^{2}$ Reproductive Biotechnology Research Center, Avicenna Research Institute, ACECR, P.O. Box: 19615-1177, Tehran, Iran.

${ }^{3}$ Research Institute of Animal Embryo Technology, Shahrekord University, P. O. Box: 115, Shahrekord, Iran.

\section{Reference}

Abolfazl Shirazi, Fatemeh Taheri, Hassan Nazari, Maryam Norbakhsh-nia, Ebrahim Ahmadi and Banafsheh Heidari. Developmental competence of ovine oocyte follow- ing vitrification: effect of oocyte developmental stage, cumulus cells, cytoskeleton stabiliser, FBS concentration, and equilibration time. Zygote. Published by Cambridge University Press, 14 August 2012. doi: 10.1017/S096719941200038X. 